\title{
Fueling the heart: Shifting the myocardial metabolome by targeting endothelial autophagy
}

\author{
Nicholas D. Andersen, MD
}

From the Department of Cardiac Surgery, Boston Children's Hospital, Harvard Medical School, Boston, Mass. Disclosures: Author has nothing to disclose with regard to commercial support.

Received for publication Aug 5, 2018; accepted for publication Aug 10, 2018; available ahead of print Sept 25, 2018

Address for reprints: Nicholas D. Andersen, MD, Boston Children's Hospital, Department of Cardiac Surgery, 300 Longwood Ave, Bader 2731, Boston, MA 02115 (E-mail: Nicholas.Andersen@cardio.chboston.org). J Thorac Cardiovasc Surg 2019;157:194-5

$0022-5223 / \$ 36.00$

Copyright (C) 2018 by The American Association for Thoracic Surgery

https://doi.org/10.1016/j.jtcvs.2018.08.012

The heart is a contractile mass in perpetual motion, and the metabolic demands of the heart exceed those of any organ in the body. Fuel for the heart is generated primarily through oxidative phosphorylation, where mitochondria, oxygen, and intracellular enzymes are used to generate adenosine triphosphate from fatty acids and carbohydrates. Of the 2, fatty acids are used in greater quantity $(\sim 70 \%$ of cardiac energy requirements), but carbohydrates are a more efficient energy substrate. For this reason, investigators have recently hypothesized that increasing glucose oxidation (at the expense of fatty acid oxidation) may improve cardiac efficiency and function in disease states with deranged myocardial bioenergetics, such as ischemia-reperfusion injury and heart failure.

In the current issue of the Journal, Altamimi and colleagues $^{2}$ explore the role of endothelial cell autophagy in regulating metabolic substrate use by the myocardium. Autophagy is the process of recycling cellular components to maintain cellular homeostasis, and prior studies from this group and others have suggested that endothelial autophagy may be important for a host of physiologic processes including metabolism. Experiments using isolated working mouse hearts demonstrated that tissue-specific inhibition of autophagy within the endothelium led to a diminished reliance on fatty acids as a fuel source in response to insulin, as well as after ischemia-reperfusion injury. In vitro studies further demonstrated that inhibiting endothelial autophagy reduced the expression of lipid chaperone fatty acid binding proteins, hinting that decreased fatty acid translocation may represent the mechanism of action. However, despite achieving a more "efficient" metabolic profile with reduced fatty acid use, there were no improvements in cardiac function with inhibition of endothelial autophagy. The authors speculate that although inhibition of endothelial cell autophagy led to what should be a more favorable metabolic profile, autophagy may play an important role in other cellular processes important for recovery from ischemiareperfusion injury. Thus, the metabolic benefits of

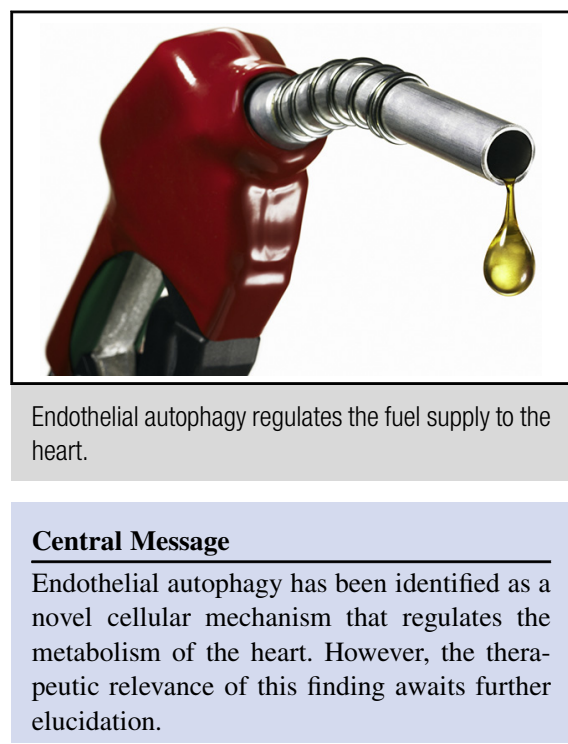

See Article page 185

inhibiting endothelial autophagy may have been offset by detriments in other areas.

The study by Altamimi and colleagues ${ }^{2}$ is notable for several reasons. Autophagy has been identified as a cellular process with a novel role in regulating metabolic substrate use of the heart, and therefore could represent a new target for therapeutic intervention. Further, the recognition that the endothelium plays a role in substrate delivery to the myocardium suggests new information about the requirements for functional endothelium for normal heart metabolism. This study provides yet another fascinating example of how deciphering the complex regulatory interplay between the endothelium and the underlying muscular tissues is critical to understanding vascular and cardiovascular biology. Despite these important findings, considerable work is needed to realize the translational potential of this study. The finding that inhibiting endothelial autophagy did not lead to functional changes in the heart suggests that unraveling the therapeutic role of autophagy (if any) will likely not be a simple task. It may be a "catch22 " of competing and contradictory cellular processes; inhibiting endothelial autophagy leads to a more favorable metabolic profile of substrate use but may deter recovery from ischemia-reperfusion injury because of the requirement for functioning autophagy systems for other important processes of cellular recovery. Thus, the road between autophagy and cardiac therapy appears somewhat obscured. 


\section{References}

1. Jaswal JS, Keung W, Wang W, Ussher JR, Lopaschuk GD. Targeting fatty acid and carbohydrate oxidation-a novel therapeutic intervention in the ischemic and failing heart. Biochim Biophys Acta. 2011;1813:1333-50.
2. Altamimi TR, Chowdhury B, Singh KK, Zhang L, Mahmood MU, Pan Y, et al. A novel role of endothelial autophagy as a regulator of myocardial fatty acid oxidation. J Thorac Cardiovasc Surg. 2019; 157:185-93. 